\title{
Inquiring and Measuring Behaviors: Unnatural Factors of Stupidity, Evil, and Mental Disorder
}

\author{
Monique Cardinal \\ University of Rhode Island, Kingston, USA \\ Email: Coralmelonsage@gmail.com
}

How to cite this paper: Cardinal, M. (2021). Inquiring and Measuring Behaviors: Unnatural Factors of Stupidity, Evil, and Mental Disorder. Open Journal of Social Sciences, 9, 295-303.

https://doi.org/10.4236/jss.2021.910021

Received: August 15, 2021

Accepted: October 24, 2021

Published: October 27, 2021

Copyright (c) 2021 by author(s) and Scientific Research Publishing Inc. This work is licensed under the Creative Commons Attribution International License (CC BY 4.0).

http://creativecommons.org/licenses/by/4.0/

\begin{abstract}
Behavioral stupidity, evil, and insanity are topics that are seen as controversial in mainstream conversations but also invoke confusion and subjectivity more than rational analysis in order to reduce confusion. For clarity on the topic, this article attends to the application of metamemetics and multilogicality, a theory proposed by Diego Fontanive to identify and analyze their characteristics. Results of this analysis suggest that more empirical work in social sciences is needed to be used toward understanding, measuring, and potentially managing, identifying, and potentially reducing or preventing disorder, evil, and stupidity if possible.
\end{abstract}

\section{Keywords}

Behavioral Psychology, Memetics, Applied Critical Thinking, Groupthink

\section{Introduction}

This essay suggests a review of distinctions, aspects, and inquiries about stupidity, evil (in the sense of profoundly immoral and unethical behaviors/intentions/ actions, and non-clinical mental disorders, which is followed by a discussion about available measures and related theories. The main goal in this study is to find a deductive, inquiring way to assess behavior and thought processes based on multilogical thinking in groups or while individually attempting to inquire about our behavior without any interference from our psychological or sociological conditioning. Another goal for this paper is to identify and understand aspects, roots, and justifications for such causes or characteristics without a biased framework of thinking.

These aspects are not derivative of/from genetic/biological factors. Another consideration is distinguishing neurological malfunction from psychological malfunction. If the psychological component is developing after birth and be- 
cause of psychosocial conditioning, is there a way to examine the behaviors and thought patterns behind them? If schizophrenia can be affected by psychological factors, not necessarily something innate or determined by neurological dysfunctions, then where might places be observed? For example, how do cult leaders become convinced they are divine or "chosen"?

Are conspiracy theorists' mindsets based on a long period of loneliness and feeling of worthlessness combined with technological freedom and without the ability and right tools to think and listen properly, duration neglect, and inattention bias? They might forget or might be aware of work behind the scenes before a researcher becomes an expert, for example. How do we relate to loneliness at the moment? How do we identify problems, such as this one before our mind builds narratives, consensus, feeling the need for a purpose or identity, and other ruts of thinking and behaving?

\section{Aspects of Behavioral Stupidity}

When exploring aspects of behavioral stupidity, it is important to make some distinctions and observations when examining these topics from a rational and multilogical perspective as opposed to how stupidity is commonly perceived. One distinction to note is that behavioral stupidity is not the same as ignorance or irrationality (see Figure 1). Irrational thinking and justifications for those thoughts, feelings, and behaviors that are unanalyzed can lead to stupidity. Ignorance that is associated with a means of denial or stubbornness and cultural imprinting can be considered metaignorance (not knowing and particularly not even caring to know how ignorant we are about something), similar to the Dunning-Kruger effect, which can lead to stupidity (stupidity as described in this paper). Suggesting other perspectives on the understanding of behavior can distinguish the reality of what is observed from being controversial or not taken seriously, as is suggested by literature on the topic.

In a survey collecting responses from 21 people, the following is a list of some aspects of stupidity that were considered relevant:

- Looking for consensus

- Persistent contradictions

- Indoctrination

- Entitlement

- Extreme monodirectional "thinking" (like radicalization, polarization, highly ideological reasoning, intolerance, and hatred driven by ideals)

- Biases (such as comparison bias, normalcy bias, halo effect, duration neglect, and inattention bias)

- Ritualization

- Desire (such as winning, looking smart, or being different/special)

- Rejection of criticism/science/intellectual approaches

- Denial (and blaming and believing in what has been denied and blamed)

- Not listening

- Not inquiring into our psychological conditioning 


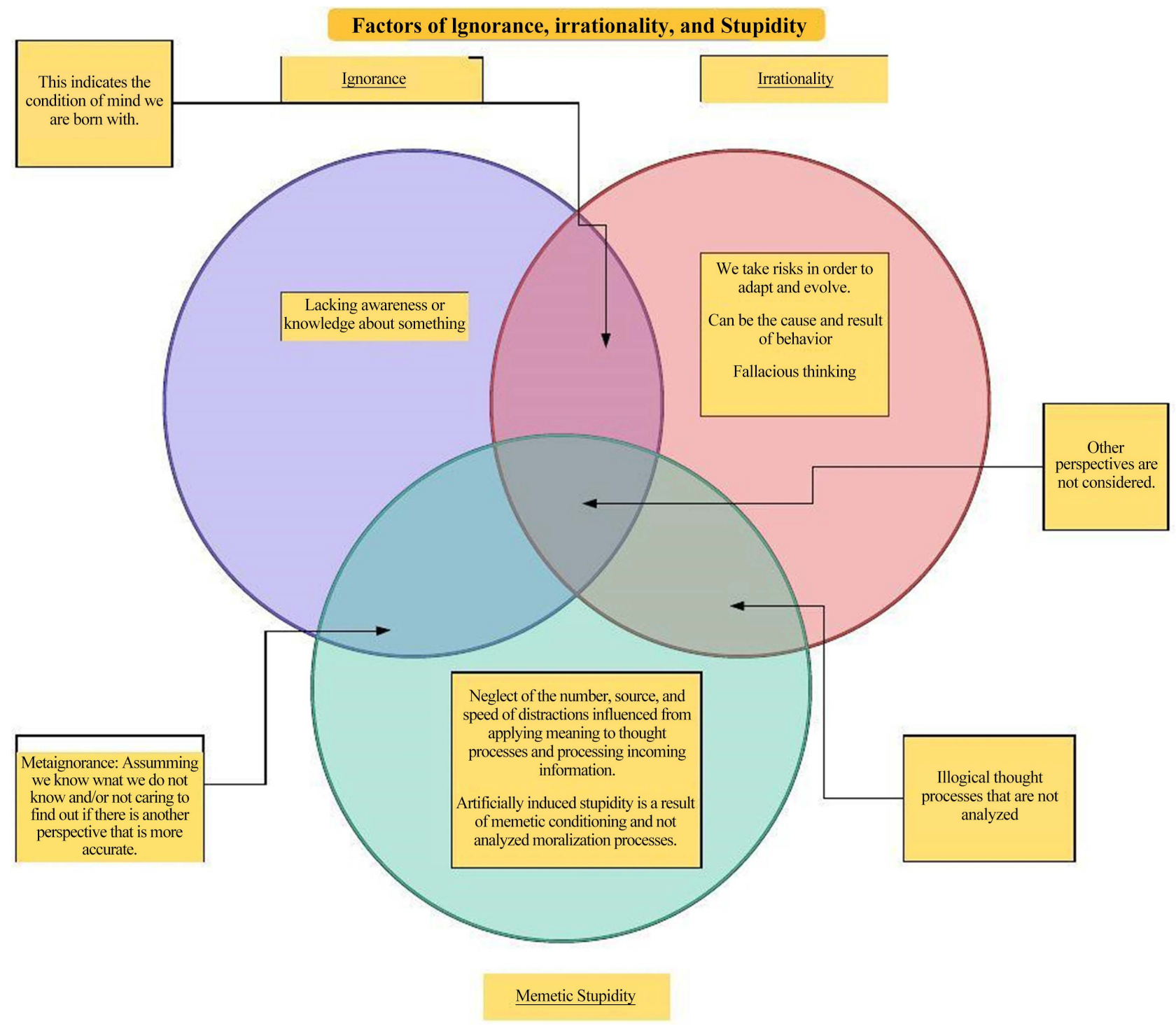

Figure 1. Differences between ignorance, irrationality, and stupidity.

- Polarization/labeling

- Framing bias (focusing almost entirely on how an information is presented instead of analyzing the information itself, its contents and premises)

- Following/groupthink without analyzing (entropic or agreeability)

- Negligence of the number of distractions

- Confusion between how things are and how we would like them to be

- Irresponsible use of freedom

- Militant ideologies

- Controlling ideas

- Clinging to ideas of a problem and a solution; therefore confusing the root of a problem with its symptoms

- Inability to identify and distinguish between/amongst myth, fact, theory, and opinion

- Amoral proclivities related to a bad relationship with natural selfishness 
- Chronic self-doubt/confidence and ignoring the quality of psychological competence while boosting confidence for emotional appeal

- Dullness/absentmindedness

- Refusing to see other perspectives

- Digital self-esteem

- Ambiguity

- Fixation/neurotic reasoning

- Excessively aggressive or positive thinking

- Machiavellian behavior/memetic competitiveness

To expand on the definition in this article, behavioral stupidity results from the number of neglected distractions that constantly prevent us from slowing down our thought processes to allow for refined thinking and questioning. The thought processes combined with in-person and online interactions and information processing speeds up and diversifies interferences.

We have diversified escape mechanisms and a memetic and digital sense of goals, identity, self-esteem, and confidence. The structure, connections, and speed of thought processes are conditioned. We read content via our ideas about morals, truth, and reality, affected and infected by the collective and personal history of psychological pictures.

Models are made for measuring aspects of evil and mental disorders. Similar to the complexity of factors to consider regarding relevant measurements, assessment of the tenacity of behavioral stupidity requires identifying considerations and issues. One of the problems with creating an equation or scheme is that the level of our conditioning can be intense, and in some cases, memeplexes can be destructive to their hosts. Controlling stupidity can be harmful in some cases (McWhorpj, 2017). Another area of complexity is distinguishing ideas of stupidity from empirical evidence about it, ergo the patterns of thinking and behavior associated with it.

Complex factors to consider in assessing stupidity include psychological conditioning via family values, personal or collective history, cultural institutions, common sense, epistemic acceptances, and factors which are non-sociological. One inquiry into possible assessment might be seeing how frequently certain aspects of each behavioral outcome occur in the context of realistic situations. How are stupidity, evil, and personality disorders analyzed scientifically or in unbiased ways, and can there be more approaches to understand the dynamics of thought processes behind them?

Another look at measuring stupidity could include tracking the degree of attentiveness, structure of thought processes and speed of our responses after reacting. If we first pause the internal dialogue before formulating thoughts and opinions based on interference via interactions, we could reduce behavior that tends to be labeled stupid and disorderly. Another measure could be the ability to separate the present self (what we associate with versus what we'd like to associate with versus what others associate us with and what we would like them to 
associate us with). These include the historical, cultural, and societal conditions that interfere with our thinking and interpretation as we engage in an experience.

\section{Aspects of Evil}

First, it is important to distinguish memetic evil from biological violence. One anthropological theory is about the killer ape by Robert Ardrey, theorizing that war and interpersonal aggression was an evolutionary drive. Hunting was a part of survival and evolution, and aggression a limbic reaction. This cannot contain thought processes and is related to adaptation (see: game theory, ecological adaptation).

A sociological theory based on empirical evidence is Phillip Zimbardo's dehumanization and deindividuation process (Zimbardo). One critique of parts of the theory could help point out details to his theory of evil also. Empirical evidence suggests following authority is conditional, that followers identify with them, and believe the leader is right. Also, conformity is unnatural and does not cause tyranny (Haslam \& Reicher, 2012). While they are aware of what they believe, followers are blind instead to anything but the belief (Haslam \& Reicher, 2012).

Here are some aspects of evil, derived from Zimbardo's theories:

- Systematic, situational, and dispositional (what is brought to the situation) evil. This includes a process of dehumanization, entropic and agreeableness of groupthink, and deindividuation

- Compulsiveness (inferring the possibility of monological thought processes)

- Impulsiveness (no forethought)

- imitating

- Groupthink (the type of GT focused on exessive agreeableness in a group [like in a cult, for instance] or the type of GT which tends to have entropic dynamics like the formation of two antagonistic sub-groups within the same group [like what happened for instance in Islam between Sunni and Shia and their difference within the same memeplex [Islam in this case])

- Ideas of a solution and ideas of a problem

- Desire to search for answers and consensus based on unanalyzed assumptions Assessing evil has been explored via theories by Milgram, Zimbardo and approaches like the Depravity Standard for judging crimes in court. Phillip Zimbardo also mentions paradoxes of time. One paradox is that people are not aware of the powerful impact time has on thoughts, feelings, and actions, also fueled by duration neglect and inattention bias. The second paradox is that someone's attitude toward time can be beneficial unless the attitude persists over others. This may be why holding a grudge or aggressive or positive thinking or forgetting details of an experience are important conditioned ways of thinking to consider when processing information. The third paradox indicates that our perspective is shaped by personal experiences, giving shape to a nation or identity (The Time Paradox). What is important is the distinction between chronological, measurable time in physics, and the psychological time, produced by 
thoughts (Personal communication with D. F, 2021).

How long does it take for thought processes to take over the act of thinking and the formation of rational awareness, and what details in these interferences and interactions are missed or misunderstood, and why? Can the noise be reduced? An example of a crucial link between evil and stupidity is related to responding to the search for answers, based on finding solutions we accept and making others agree about in order to confirm our own beliefs.

Relevant literature has been written about how memes that kill their hosts, theories could be made about the intensity of the memes' impact, factors of inward and outward acts of violence, and consequences due to preferring to serve ideas over rationalizing through delusions (see: Table 1). One question for inquiry could be into how we relate to cycles of destruction and creation as well as memetic identities like victimhood and heroism. Another inquiry could be made in regard to becoming and choosing something to identify with along with something to identify as antagonistic rather than understanding the various conditionings and the idea that we deserve to pick one over another, for example, because we may want to be different.

A final example an inquiry regarding how preceding thoughts and associating memes can lead to violence, can there be a link amongst mental disorders, stupidity, and evil?

For example: one cultural aspect that might get ignored in discussion about sociology and respect for artifacts is that everyone's thinking style is different because we come from different consulting and backgrounds. It's an example of an internal action which could contribute to violence.

\section{Aspects of Non-Clinical Mental Disorder}

This section is based on the book, Genes, Memes, Culture, and Mental Illness by Hoyle Leigh. First, it is important to distinguish neurological malfunctions from psychological malfunctioning that lead to mental disorders. Neurological factors include diseases like Parkinson's disease, Alzheimers, Cerebral Palsy, Multiple Sclerosis, and more, but psychological factors include adverse childhood experiences, personality traits, genetic predisposition, among other factors.

Here is a list of some of the aspects of psychological malfunctions leading to mental disorders:

- Extreme is happening all the time

- Delusions/obsessions/neuroses

- Rituals

- Obsession

- Compulsiveness

- Inattentiveness

- Naivety + gullibility

- Devotion

- Excess and extreme behavior patterns (borderline-alike for instance or merely pivoted in emotional immaturity) 
- Inability to adapt

- Imprecision

- Lack of introspection

- Disorganized thoughts

These aspects are associated with describing syndromes listed in Table 2.

What therapies related to understanding thoughts are available and effective?

Dialectical, cognitive-behavioral, interpersonal, psychodynamic, and rational emotive behavior are mentioned in Leigh's textbook. Therapies that are not focused on understanding thoughts include massage therapy, mediation, bibliotherapy, electroconvulsive therapy, exercise, sleep, and sedation (Leigh, 2011).

Memetics helps lay out the mechanics of how social environments will affect our brains and vice versa (278, Leigh, 2011). Leigh highlights the importance of analyzing connections made between and among perceived environment, memory, and genes, and interaction in the real environment. Parts of the mind may reject or consent to self plexes/meme-leaders. When the brain lacks sufficiently powerful reasoning ability (the ability to manipulate the memes), or when competing memes are intransigent and people are unable to reach an agreed-upon course of action based on compromise, then the conflict becomes irresolvable and severe anxiety issues arise (Leigh, 2011).

Table 1. Categories of violence.

\begin{tabular}{|c|c|}
\hline Type of Violence Act & Memetic Goal and Mode of Function \\
\hline Conflict & $\begin{array}{l}\text { Minimal to zero memetic goal and functioning. When it is memetic, it stems } \\
\text { from an idea of feeling safe, or ideas and values attached to biological needs. }\end{array}$ \\
\hline Wars & $\begin{array}{l}\text { An idea of an opponent is created, a weaponized assurance of belonging and } \\
\text { safety. Usually, opponents agree to fight and while doing so they will } \\
\text { accomplish a goal. The idea of a need is confused with the actual need. }\end{array}$ \\
\hline Genocide & $\begin{array}{l}\text { A group of people have an idea about replacing and dissolving opposing } \\
\text { groups of ideas and their hosts. The hosts are deprived of resources and } \\
\text { cultural artifacts and chance of defense. }\end{array}$ \\
\hline Genocide & $\begin{array}{l}\text { The host has an idea that a person will implement via killing and while } \\
\text { submitting to the idea of taking their own life to justify a meme. }\end{array}$ \\
\hline
\end{tabular}

Table 2. Adapted from Leigh, Hoyle. Genes, Memes, Culture, and Mental Illness. pp. 223-227 (Leigh, 2011).

\begin{tabular}{ll}
\hline $\begin{array}{l}\text { Irrational fear-anxiety-depression } \\
\text { Note: neurotic depression and anxiety are } \\
\text { mainly caused by developmental hangups } \\
\text { and faulty learning (Leigh, 2011). }\end{array}$ & $\begin{array}{l}\text { Anxiety, panic, ASD, depression (neurotic versus } \\
\text { syndromic), BPD, HPD, NPD, mania, adjustment } \\
\text { disorders, avoidance traits and personalities, phobias }\end{array}$ \\
\hline Attention-cognition & $\begin{array}{l}\text { Delirium, dementia, impulse control, ADHD, OCD } \\
\text { traits, antisocial traits }\end{array}$ \\
Reality-perception & $\begin{array}{l}\text { Psychosis, dissociation, conversion, somatoform, } \\
\text { somatization, misattribution }\end{array}$ \\
Pleasure-motivation & $\begin{array}{l}\text { Substance use and abuse, addiction to substances, } \\
\text { addiction to beliefs, fanaticism }\end{array}$ \\
Primary Memetic syndromes & $\begin{array}{l}\text { Eating disorders, facetious syndromes, malingering, } \\
\text { meme-directed irrational acts }\end{array}$ \\
\hline
\end{tabular}




\section{Conclusion}

We have defined three non-biological behavioral patterns: stupidity, evil, and mental disorder. This paper lacks necessary primary resources and empirical research into understanding behaviors in new ways. There is complexity in measuring disorder, evil and stupidity. The goal is to identify, assess, and understand a disordered way of thinking.

One recommendation for enhancing the significance of this study is seeing which characteristics of stupidity in a given control group, such as an online environment, are most relevant, what is common, why, and how to counteract the consequences. For example, if people are competing to look smart or win an argument, counteracting this with a rational way to absorb information may prevent confusion and unnecessary anger, reactivity that may be seen in social media groups. Another method may be to create algorithms to direct users toward accurate information or less biased approaches. What is needed is more quantitative research in regard to measuring and preventing consequences of artificial stupidity, in particular, and an increase in complexity because we are living in an era of globalization, converging replicator point and space age.

Lastly, finding available models for evil, stupidity, and mental disorders in this paper might be possible. For example, there are studies about distractedness and duration neglect impacting judgement.

\section{Acknowledgements}

I wanted to express appreciation for help from anonymous reviewers and editors, and Diego Fontanive for permission to use him as a resource for information.

\section{Conflicts of Interest}

The author declares no conflicts of interest regarding the publication of this paper.

\section{References}

Haslam, S. A., \& Reicher, S. D. (2012). Contesting the "Nature" of Conformity: What Milgram and Zimbardo's Studies Really Show. PLoS Biology, 10, e1001426. https://doi.org/10.1371/journal.pbio.1001426

Leigh, H. (2011). Genes, Memes, Culture, and Mental Illness: Toward an Integrative Model. Springer. https://doi.org/10.1007/978-1-4419-5671-2

McWhorpj, P. (2017, February 18). The Seven Degrees of Stupid. YouTube. https://www.youtube.com/watch?v=r3 JLAAck2Q

The Time Paradox (n.d.). https://www.thetimeparadox.com/

Wikimedia Foundation (2021, July 4). Memeplex. https://en.wikipedia.org/w/index.php?title=Memeplex\&oldid $=1031851708$

Zimbardo, P. (n.d.). The Psychology of Evil. TED.

http://www.ted.com/talks/philip zimbardo the psychology of evil 


\section{Definitions}

Behavioral/Memetic Stupidity: An acquired way of reacting that tends to lead to the individual having erroneous thinking and absence of thinking differentiating from irrationality or ignorance, which may be worse than bad/monological thinking: thinking through beliefs that malevolently condition the ontological, epistemic and axiological ability to generate thought processes.

Evil: As a meme, for this article's context, it is a learned way to act that leads to systematically preventing healthy symbiosis in the sense of inter-relationships, communication and mutual understanding both in the psychological and sociological life. This definition comes from a pattern of mode to control people in order to fulfill an idea of what a problem and solution are if given the opportunity. Neurological disorders that may lead to actions that are called evil do not indicate the memetic components or imply that evil is enjoyed. There is a lack of thinking skills and inherent training capable of influencing (positive) thought process and reactions, therefore behaviors and actions.

Disorder: "Mental disorders (to be seen separately from mental illnesses) are conditions that affect your thinking, feeling, mood, and behavior. They may be occasional or long-lasting (chronic); (Medline)."

Memetics: The study of how memes (values, epistemically acceptances, memories and how we interpret them, ideas of expectations, norms, taboos, etc.) work.

Memeplex: Group of memes, like a belief system, in someone or within a group. See: The Selfish Gene by Richard Dawkins (1976).

Metamemetics: Diego Fontanive's adaptation of Memetics, studying the (Wikimedia Foundation, 2021) malevolent/deviating/problematic impact that certain memes have on our psychology (therefore often collectively as well) and the way we organize our awareness (intended as 1) The brain's ability to pay attention combined with 2) The brain's ability to create thinking models). "It also involves the decoding the validity or invalidity of the premises behind the meaning of the memes to understand whether the premises are valid or not (personal communication with D. F., 2020).”

Multilogical thinking: Different ways of reasoning so as to also form models of awareness (awareness as described in the point above), such as lateral, dynamic, analytical, and applying via negativa, finding information (e.g. a theory) that purposely contradicts what you think about something.

Psychological pictures (coined by Diego Fontanive): Memes that interfere with thinking and perception in the mind (therefore with the formation of awareness) when we associate thoughts with our thinking and interactions with people on a constant basis (personal communication with D. F., 2020). This is not to be confused with the Psychiatric term: psych images. 